\title{
Contrast-Enhanced Ultrasound Imaging for the Detection of Focused Ultrasound-Induced Blood-Brain Barrier Opening
}

\author{
Ching-Hsiang Fan¹, Wun-Hao Lin, Chien-Yu Ting1, Wen-Yen Chai², Tzu-Chen Yen³, Hao-Li Liu ${ }^{2}$ and \\ Chih-Kuang Yeh ${ }^{\bowtie}$
}

1. Department of Biomedical Engineering and Environmental Sciences, National Tsing Hua University, No. 101, Section 2, Kuang-Fu Road, Hsinchu, Taiwan 30013;

2. Department of Electrical Engineering, Chang-Gung University, 259 Wen-Hwa 1st Road, Kuei-Shan, Tao-Yuan, Taiwan 33302;

3. Department of Nuclear Medicine and Molecular Imaging Center, Chang Gung University and Memorial Hospital, No. 5, Fu-Shing Road, Kuei-Shan, Tao-Yuan, Taiwan 33305.

\begin{abstract}
$\triangle$ Corresponding author: Department of Biomedical Engineering and Environmental Sciences, National Tsing Hua University, No. 101, Section 2, Kuang-Fu Road, Hsinchu, Taiwan 30013, R.O.C. Tel: +886-3-571-5131, ext. 34240; Fax: +886-3-571-8649 (C.-K. Yeh). Department of Electrical Engineering, Chang-Gung University, 259 Wen-Hwa 1st Road, Kuei-Shan, Tao-Yuan 33302, Taiwan, R.O.C. Tel: +886-3-211-8800, ext. 5677; Fax: +886-3-211-8026 (H.-L. Liu). E-mail address: ckyeh@mx.nthu.edu.tw (C.-K. Yeh), haoliliu@mail.cgu.edu.tw (H.-L. Liu).
\end{abstract}

() Ivyspring International Publisher. This is an open-access article distributed under the terms of the Creative Commons License (http://creativecommons.org/ licenses/by-nc-nd/3.0/). Reproduction is permitted for personal, noncommercial use, provided that the article is in whole, unmodified, and properly cited.

Received: 2014.05.05; Accepted: 2014.07.10; Published: 20I4.08.0I

\begin{abstract}
The blood-brain barrier (BBB) can be transiently and locally opened by focused ultrasound (FUS) in the presence of microbubbles (MBs). Various imaging modalities and contrast agents have been used to monitor this process. Unfortunately, direct ultrasound imaging of BBB opening with MBs as contrast agent is not feasible, due to the inability of MBs to penetrate brain parenchyma. However, FUS-induced BBB opening is accompanied by changes in blood flow and perfusion, suggesting the possibility of perfusion-based ultrasound imaging. Here we evaluated the use of MB destruction-replenishment, which was originally developed for analysis of ultrasound perfusion kinetics, for verifying and quantifying FUS-induced $B B B$ opening. MBs were intravenously injected and the $\mathrm{BBB}$ was disrupted by $2 \mathrm{MHz}$ FUS with burst-tone exposure at 0.5-0.7 MPa. A perfusion kinetic map was estimated by $M B$ destruction-replenishment time-intensity curve analysis. Our results showed that the scale and distribution of FUS-induced BBB opening could be determined at high resolution by ultrasound perfusion kinetic analysis. The accuracy and sensitivity of this approach was validated by dynamic contrast-enhanced MRI. Our successful demonstration of ultrasound imaging to monitor FUS-induced BBB opening provides a new approach to assess FUS-dependent brain drug delivery, with the benefit of high temporal resolution and convenient integration with the FUS device.
\end{abstract}

Key words: focused ultrasound, blood-brain barrier, microbubbles, destruction- replenishment, dynamic contrast-enhanced MRI.

\section{Introduction}

The blood-brain barrier (BBB) is a specialized structure in the central nervous system (CNS) that consists of cerebral capillary endothelial cells, basal lamina and glial processes [1-3]. These layered cell structures are critical for maintaining brain homeostasis by forming tight junctions with low permeability to control the passage of molecules between the blood and brain parenchyma $[3,4]$. However, the BBB 
also hinders transport of therapeutic agents such as drugs, genes or antibodies from blood into brain tissue, thus lowering treatment efficiency $[5,6]$. Burst-tone focused ultrasound (FUS) exposure in the presence of microbubbles (MBs) has been shown to transiently open the BBB to allow free penetration of therapeutic molecules into the brain. Due to its unique strengths to change BBB integrity in a noninvasive, transient, and localized manner, FUS-induced BBB opening has been recognized as a potentially promising approach for brain drug delivery $[7,8]$.

Several in vivo imaging techniques have been used to detect FUS-induced BBB opening. Among them, the most consistent and reliable approach has been to intravenously (i.v.) inject an imaging contrast agent followed by detection of the contrast change by magnetic resonance imaging (MRI). For example, some of the first experiments involved administration of gadolinium diethylenetriamine pentaacetic acid (Gd-DTPA; $937 \mathrm{Da}$ ) for the detection of BBB opening by contrast-enhanced T1-weighted MRI [7, 8]. Magnetic nanoparticles have also been used to monitor FUS-induced BBB opening by $\mathrm{T} 2-$ or $\mathrm{T} 2{ }^{*}$-weighted MRI [9-11]. When using Gd-DTPA or magnetic nanoparticles, the concentration of leaked contrast agent can be accurately quantitated by measuring spin-spin or spin-lattice relaxivity $[11,12]$. Dynamic contrast-enhanced (DCE)-MRI with compartment modeling was also recently used to evaluate contrast agent kinetics and the change in CNS permeability after induction of BBB opening [13, 14]. Other methods besides MRI have been used to detect FUS-induced BBB opening, such as single photon emission computed tomography (SPECT) with i.v. administration of $99 \mathrm{mTC}$-DTPA radiotracer as the contrast agent, or small-animal photoacoustic imaging with gold nanorods $[15,16]$. In addition, dynamic changes in microvasculature during FUS-induced BBB opening have been microscopically observed by fluorescent multi-photon microscopy, and with a high-speed camera $[17,18]$.

Ultrasound has long been utilized for diagnostic and imaging purpose. Using ultrasound to monitor FUS-induced BBB opening provides several advantages including no requirement for radiation or compatibility concerns, and high temporal resolution which allows real-time monitoring. Furthermore, therapeutic and imaging ultrasound excitations can be integrated into a single ultrasound platform with overlapping aperture and intrinsically co-localized setup. Goertz et al. first attempted monitoring FUS-induced BBB opening by determining the time-intensity curve (TIC) change of concurrent clinical ultrasound imaging (9-17 MHz) [19]. Unfortunately, this study did not provide strong evidence of a correlation between FUS-induced BBB opening and the TIC. We similarly reported that BBB opening could not be successfully monitored by ultrasound imaging $(25 \mathrm{MHz})$ in a small-animal model, although red blood cell (RBC) extravasations were detectable in real-time under excessive applied pressure [20]. Under these conditions, the RBCs provided sufficient backscattered signal for contrast-enhanced ultrasound imaging. A major reason for the failure of contrast-enhanced ultrasound imaging in monitoring FUS-induced BBB opening is that MBs are designed to be in the micrometer size range to maximize their echogenic capability. Their large size limits the location of MBs to the vascular/ microvascular system, and they do not leak into CNS parenchyma. MB-enhanced ultrasound imaging to detect FUS-induced BBB opening is thus intrinsically challenging.

An alternative may be provided by monitoring the dynamics of $\mathrm{MB}$ destruction-replenishment (D/R), which was originally developed for measuring perfusion kinetics in ultrasound imaging. High ultrasound pressure can cause destruction of i.v. administered, circulating MBs within the imaging zone. The resulting transient drop in signal as well as the dynamics of signal re-appearance can be deduced from the ultrasound imaging TIC. These MB D/R dynamics are known to be highly correlated with vascular dynamics and perfusion kinetics. Wei et al. first proposed the use of MB D/R to quantify myocardial blood flow [21]. In 2001, Rim et al. pioneered the use of $\mathrm{MB} \mathrm{D} / \mathrm{R}$ to estimate changes in cerebral microvascular blood volume and mean MB velocity in an in vivo animal model [22]. Since local FUS-induced $\mathrm{BBB}$ opening involves a change in vascular blood-brain permeability, the dynamics of local blood flow may also be affected, suggesting the possibility of using the ultrasound-imaging $\mathrm{D} / \mathrm{R}$ approach to monitor FUS-induced BBB opening.

The aim of this study was therefore to evaluate whether MB D/R-based perfusion kinetics could be used to monitor FUS-induced BBB opening by ultrasound imaging. We hypothesized that FUS-induced BBB opening induced a change in perfusion kinetics, and the measurement of this change by ultrasound imaging could reflect the success in monitoring of FUS-induced BBB opening.

\section{Materials and Methods}

\section{MB Destruction-Replenishment (D/R) Model}

Previous studies suggested that blood flow and vasculature dynamics can be evaluated by MB disruption and replenishment, using a growing mono-exponential model or sigmoid function ap- 
proximation [21, 23-25]. Yeh et al. subsequently proposed a modified sigmoid function to improve the estimation precision from the TIC (Figure 1(a)) [26]. The replenishment cycle is estimated from the received ultrasound B-mode signal using a modified sigmoid function, as follows:

$$
R(t)=A\left(\frac{1}{1+e^{-\alpha(t-c)}}\right)
$$

where $R(t)$ is the received intensity after replenishment of MBs following time $(t)$; $A$ is the echo intensity of steady state (i.e., refilled MBs); $a$ is the rate constant of the sigmoidal function which is proportional to the mean blood flow velocity; and $c$ is the time interval from the start to the inflection point in the sigmoid curve.

Replenishment with intact MBs is assumed to begin at the boundary of the $-6 \mathrm{~dB}$ ultrasound beam width (i.e., $E / 2$ ) of the imaging probe. The time-intensity relationship corresponds to the inflection point of the sigmoidal function when the concentration of MBs is restored to $50 \%$ in the sample volume (i.e., at the $t_{1}$ time point). The slope of the TIC increases with time before the inflection point because of the increasing intensity of the ultrasound beam, and decreases thereafter. The flow velocity $(v)$ can be estimated as $(E / 2) / c$. The tangent slope at the inflection point $(t=c)$ can be described as $(A a) / 4$. As shown in Figure 1(a), the slope is also equal to $(A / 2) / c$, and

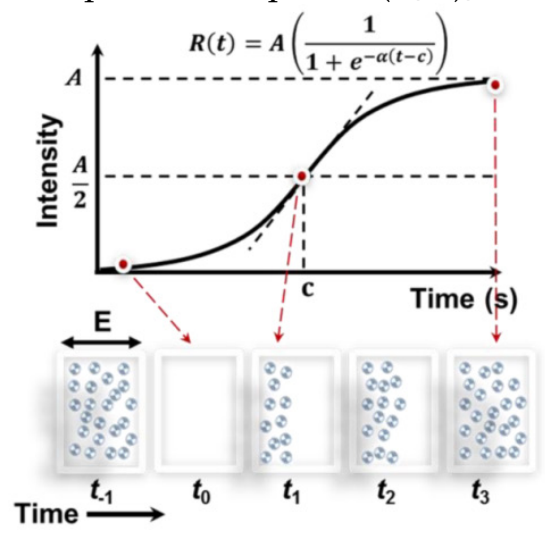

(a)

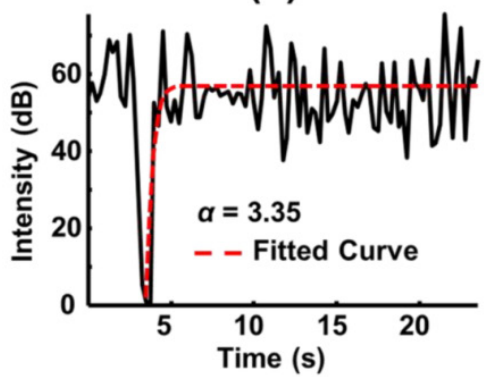

(c) then $c=2 / a$. The blood flow velocity can then be written as $(a / 4) E$. When $E$ is a constant, a linear relationship between the blood flow kinetics which is characterized by rate constant $a$ (with the unit of $\mathrm{s}^{-1}$ ) can be expected [26].

\section{Signal Processing and $\alpha$ Map Creation}

A two-dimensional (2-D) map of the $\alpha$ distribution was created to identify the change in MB replenishment intensity, and thus assess the vascular perfusion kinetic change. The intensities of MB signals in $\mathrm{D} / \mathrm{R}$ images were obtained by averaging all of the pixels within the region of interest (ROI) using a sliding window, and was presented over time to construct the TICs of MBs (Figure 1(b)). To reduce the interference of noise on TICs, we used an integrated TIC technique to reduce the effect of $a$ variability on the blood flow estimation [26]. The integrated TICs were fitted using the built-in nonlinear least algorithm of MATLAB (MathWorks Inc., MA, USA) (Figure $1(\mathrm{c})$ ), and rate constant $a$ maps were created by calculating the $a$ values from TICs of each pixel (Figure 1(d)). The $a$ values were expected to be proportional to the flow velocity, therefore the rate constant $a$ maps should display the corresponding flow velocity distribution. Finally, the rate constant a map acquired before FUS sonication was subtracted from that obtained after sonication to derive a rate constant a-change map.

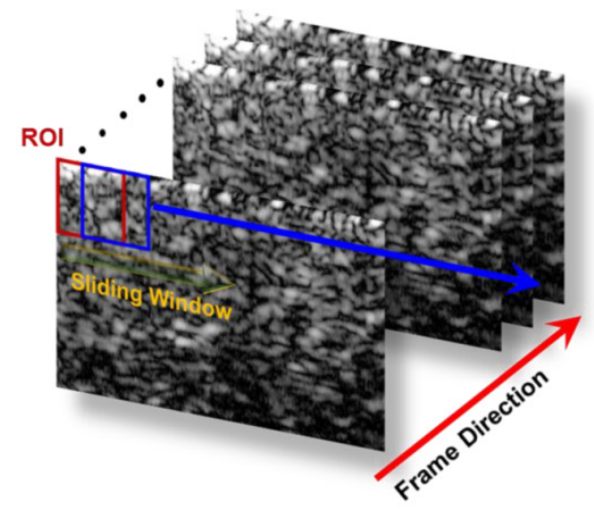

(b)

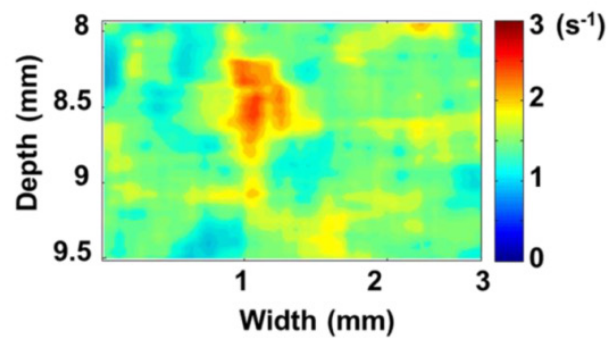

(d)

Figure I. (a) Conceptual diagrams of MB D/R approach for monitoring FUS-induced BBB opening. (b) Sequentially acquired ultrasound B-mode images. (c) TIC obtained from a specific ROI of B-mode images and estimation of rate constant $\alpha$ value. (d) Corresponding rate constant $\alpha$-mapping distribution. 


\section{Preparation of MBs}

MBs were prepared by the thin-film hydration method [20]. For a $1 \mathrm{~mL}$ formulation, 1,2-Distearoylsn-glycero-3-phosphocholine (DSPC) (Avanti Polar Lipids, AL, USA) and 1,2-distearoyl-sn-glycero3-phosphoethanolamine-N-[methoxy(polyethylenegl ycol)-2000] (DSPE-PEG-2000) (Avanti Polar Lipids) were mixed at a molar ratio of 6:1 in chloroform, dried for over $24 \mathrm{~h}$ under reduced pressure with a rotary evaporator (Rotavapor R-210, Büchi Labortechnik AG, Flawil, Switzerland) to form a lipid-film, and stored at $-20^{\circ} \mathrm{C}$. To prepare the aqueous lipid solution, the film was hydrated with $5 \mu \mathrm{L} / \mathrm{mL}$ glycerol phosphate-buffered saline (PBS, $\mathrm{pH}$ 7.4) and the gas was replaced with perfluoropropane $\left(\mathrm{C}_{3} \mathrm{~F}_{8}\right)$. Finally, the solution was intensely shaken by an agitator for $45 \mathrm{~s}$ to obtain the MB suspension. The MBs were counted and sized using a coulter counter equipped with a 30 $\mu \mathrm{m}$ sensor orifice (Multisizer 3, Beckman Coulter, Miami FL, USA) for a 0.7-20 $\mu \mathrm{m}$ range. The mean size and concentration of MBs were $1.18 \pm 0.01 \mu \mathrm{m}$ and $(35.74 \pm 0.49) \times 10^{9} \mathrm{MB} / \mathrm{mL}$ (post MBs preparation 30 min, $37^{\circ} \mathrm{C}$ ), respectively. (Additional file 1: Supplemental Figure S1-S2).

\section{Animal Preparation}

The experimental protocol of this study was approved by the Institutional Animal Care and Use Committee of National Tsing Hua University and adhered to the experimental animal care guideline (IACUC approval number: NTHU10044). A total of 28 pathogen-free adult male Sprague-Dawley rats (300-350 g) were anesthetized by chloral hydrate (400 $\mathrm{mg} / \mathrm{kg}$ ). Before the start of an experiment, rats were subjected to craniotomy and venous cannulation. The cranial window (nearly $10 \mathrm{~mm} \times 4 \mathrm{~mm}$ ) was created with a high-speed drill to reduce attenuation and distortion of the ultrasound beam. The catheter was placed into the jugular venous arch for injection of MBs and Evans Blue (EB) dye during experiments.

\section{In-House Built High Frequency Ultrasound Imaging /FUS Sonication Platform and Experiments}

The integrated platform consisted of a single-element circular-aperture FUS transducer with a hole in its center for the ultrasound imaging transducer. The outer transducer was spherically-focused annulus with a central frequency of $2 \mathrm{MHz}$ and a -6 $\mathrm{dB}$ fractional bandwidth of $83.9 \%$ (model V3966, Panametrics, MA, USA; outer element size $=26.1 \mathrm{~mm}$, inner element size $=9.9 \mathrm{~mm}$, focal length $=26.8 \mathrm{~mm}$ ), providing the high power energy needed to both destroy MBs (0.3-1.0 MPa; burst length = 10 cycles; PRF $=4000 \mathrm{~Hz}$; exposure time $=0.5 \mathrm{~s}$; mechanical index:
0.21-0.71) and generate the excitation pulse for $\mathrm{BBB}$ opening $(0.5 / 0.7 \mathrm{MPa}$; burst length $=2000$ cycles; PRF $=1 \mathrm{~Hz}$; exposure time $=60 \mathrm{~s}$; mechanical index: $0.35 / 0.50)$. The FUS transducer was driven by a function generator (HP 33120, Hewlett-Packard, TX, USA) through a power amplifier (A150, E\&I, NY, USA). The electric impedance within the transducer was matched to the output impedance of the power amplifier by an external impedance-matching circuit.

The inner transducer was customized to have a central frequency of $40 \mathrm{MHz}$ with a $-6 \mathrm{~dB}$ fractional bandwidth of $94 \%$ (element size $=3 \mathrm{~mm}$, focal length = $9 \mathrm{~mm}$ ) for acquiring ultrasound B-mode images. The arbitrary waveform generator (AWG-2040, Tektronix, CA, USA) provided 3 cycle $40 \mathrm{MHz}$ sinusoid excitation through the power amplifier (325LA, Electronic Navigation Inc., NY, USA) to the imaging transducer. The acoustic pressure of $40 \mathrm{MHz}$ imaging pulse was $2.4 \mathrm{MPa}$ (mechanical index $=0.38$ ). To prevent echoes from the destruction pulses from reaching the imaging transducer, a delay generator (DG-535, Stanford Research system, CA, USA) was used for separating imaging and destruction into two backscattered echo signals. Radio-frequency (RF) echoes were received using the same transducer through a diplexer circuit (model DIP-3, Matec Instruments NDT, MA, USA). The received RF echoes were magnified by the preamplifier (model AU-1114, MITEQ Inc., NY, USA), digitized at a sampling rate of 400 Msamples/s by the PC-based 12-bit analog-to-digital board (model CS12400, Gage Applied Tech Inc., IL, USA), and stored in a PC for generating B-mode images with MATLAB.

To achieve uniform MB destruction throughout the entire imaging plane, the $2 \mathrm{MHz}$ FUS and $40 \mathrm{MHz}$ imaging transducers were positioned together on the piezoelectric-type motor and mechanical scanning was performed (stepping resolution $=8 \mu \mathrm{m}$, traveling distance $=3 \mathrm{~mm}$, lead transmission speed $=27.5$ $\mathrm{mm} / \mathrm{s}$ ). These settings were equivalent to eight imaging frames per second with a traveling time of 0.125 $\mathrm{s}$ for each imaging region, and the FUS exposure covering the entire imaging region (Detailed imaging and FUS exposure parameters are listed in Additional file 1: Supplemental Table S1). The pressures reported in this study were calculated by measuring the peak-rarefactional pressure amplitudes with a polyvinylidene-difluoride-

type (PVDF) hydrophone (model HGL-0085, ONDA, Sunnyvale, CA, USA; calibration range $=1-40 \mathrm{MHz}$ ) in a tank filled with distilled and degassed water.

The reliability of MB D/R to estimate perfusion kinetics was tested in vitro with a vessel-phantom and in vivo in rat brain via the in-house built high frequency ultrasound imaging system (Additional file 1: 
Supplemental Figure S3) [26]. The vessel was constructed by embedding a polyethylene tube with an outer diameter of $1 \mathrm{~mm}$ before the phantom solidified and removing it immediately after. The phantom was prepared from $2 \mathrm{~g}$ of agarose powder (UltraPure ${ }^{\mathrm{TM}}$ Agarose, CA, USA) in $100 \mathrm{~mL}$ of water. The solidified phantom was immersed in a tank filled with degassed water. The flow rate of the flow chamber was set to 6 $\mathrm{mL} / \mathrm{hr}$ (i.e., $1.58 \mathrm{~mm} / \mathrm{s}$ mean velocity) with a syringe pump (KDS100, KD Scientific, PA, USA). The MBs were diluted 2000 times with $0.9 \%$ normal saline.

The feasibility of our proposed method to in vivo monitor FUS-induced rat cerebral perfusion kinetic changes $(N=3)$ at each time point (before and $5 \mathrm{~s}$ after BBB opening) was primarily tested using the same integrated platform for ultrasound imaging and FUS sonication. The center of the FUS focal zone was placed at $3.5 \mathrm{~mm}$ posterior and $2.5 \mathrm{~mm}$ lateral to the bregma (left brain), and $3 \mathrm{~mm}$ below the skull surface (cerebral cortex). During the experiments, the MBs were diluted 2000 times with $0.9 \%$ normal saline and injected into the rats by syringe pump with a constant flow rate of $10 \mathrm{~mL} / \mathrm{hr}$ (i.e., $2.63 \mathrm{~mm} / \mathrm{s}$ mean velocity). The total infusion time was $8 \mathrm{~min}$. The total infusion volume was $1.3 \mathrm{~mL}$. In order to remove brain tissue signals, the acquired B-mode images were subtracted from the image obtained without MBs injection. The subtracted images were used to construct TIC curves and calculate the $\alpha$ values for each ROIs and eventually create the $\alpha$ maps.

\section{Commercial Ultrasound Imaging System and FUS Sonication Setup}

The major in vivo animal experiments were conducted by using a high frame rate commercial ultrasound imaging system (Additional file 1: Supplemental Figure S4(a); Vevo2100, VisualSonics, Toronto, Canada;). The B-mode images were obtained by the imaging system via a $21-\mathrm{MHz}$ linear array transducer (MS250, VisualSonics) (power: 5\%; frame rate: $30 \mathrm{fps}$; lateral resolution: $165 \mu \mathrm{m})$. MB destruction was also carried out with this imaging system by operation in contrast mode with a destruction burst at $100 \%$ power. BBB opening was conducted with the same $2 \mathrm{MHz}$ FUS transducer (Detailed sonication and imaging parameters are listed in Additional file 1: Supplemental Table S2). The two transducers were arranged in parallel, separated by approximately 49 $\mathrm{mm}$ using an in-house made holder which ensured that the foci of the FUS beam and the $21 \mathrm{MHz}$ imaging beam were fixed at the same depth. The holder was mounted on a three-dimensional (3-D) motion stage. Animals were randomly divided into three groups: (1) Control group without FUS exposure $(N=7)$; (2) 0.5 MPa FUS exposure (burst length $=1 \mathrm{~ms}, \mathrm{PRF}=1 \mathrm{~Hz}$, exposure time $=60 \mathrm{~s}$, mechanical index $=0.35 ; N=9$ ); and (3) 0.7 MPa FUS exposure (same exposure conditions as group 2, mechanical index $=0.50 ; N=9$ ).

Prior to FUS exposure to induce BBB opening, a $D / R$ image series (frame rate $=30 \mathrm{fps}$, acquisition time $=20 \mathrm{~s}$; power $=5 \%$ ) was acquired, with the imaging transducer also serving to destroy MBs (10 cycle short burst excitation for $2 \mathrm{~s}$; power $=100 \%$ ). Afterwards, the process of BBB opening was conducted (at this time, the FUS transducer was repositioned, within a minute, to the target site by the 3-D motion stage). Three series of $D / R$ images were then acquired at 60, 90 and $120 \mathrm{~s}$ after BBB opening. MBs were perfused by the syringe pump with a flow rate of 10 $\mathrm{mL} / \mathrm{hr}$ to obtain $\mathrm{D} / \mathrm{R}$ images at each time point. The total infusion time was $8 \mathrm{~min}$ and the infusion volume was $1.3 \mathrm{~mL}$. In TIC estimation, B-mode images subtracted by baseline (image before $\mathrm{MB}$ injection) were performed to feature out MB-related signal intensity change. The estimated TICs were then employed to estimate the corresponding $\alpha$ values (described in above section of Signal Processing and $\alpha$ Map Creation). Detailed experimental time line of the in vivo animal experiments is shown in Additional file 1: Supplemental Figure S4 (b).

\section{MRI Verification}

After conducting FUS-induced BBB opening and $\mathrm{D} / \mathrm{R}$ sequence acquisition, animals were repositioned in the MR scanning bore for MRI (7T, ClinScan 70/30 USR, Bruker; time lapse controlled to be less than 5 min after FUS exposure), with immediate bolus administration of Gd-DTPA (Agnevist, Bayer Healthcare, $0.5 \mathrm{ml} / \mathrm{kg}$ ). DCE-MRI was performed to calculate MR permeability maps by acquiring a 3-D FLASH T1-weighted sequence $(\mathrm{TE} / \mathrm{TR}=0.76 \mathrm{~ms} /$ $2.31 \mathrm{~ms}$; slice thickness $=0.8 \mathrm{~mm}$; flip angles $=50^{\circ} /$ $200^{\circ}$; matrix size $=192 \times 132$; acquisition time $=10$ min; number of slices $=6$; FOV $=34 \times 40 \mathrm{~mm}$ ). Permeability information was obtained based on data post analysis using the Extended-Kety model to generate blood-brain permeability mapping (i.e., Ktrans mapping) [27].

\section{Histological Examination}

EB dye $(100 \mathrm{mg} / \mathrm{kg}$; $960 \mathrm{Da}$; forms a $67 \mathrm{kDa}$ complex with serum albumin) was administered 5 min prior to the process of BBB opening, to verify its success. Animals were sacrificed $10 \mathrm{~min}$ after completing the experimental procedure, and perfused with $0.9 \%$ normal saline via the left cardiac ventricle until colorless perfusion fluid appeared from the right atrium. The brain tissues were removed, and fixed with $10 \%$ neutrally buffered formalin (Sigma-Aldrich) followed by embedding in optimal cutting tempera- 
ture compound (Tissue-Tek OCT, Sakura Finetek Inc., Torrance, CA, USA) and stored at $-50^{\circ} \mathrm{C}$. Histological brain sections were obtained with a Cryostat Microtome at a slice thickness of $10 \mu \mathrm{m}$ in the same direction as ultrasound imaging. BBB opening was identified by the leakage of EB dye into brain tissues. Hematoxylin and eosin (H\&E) staining was applied to confirm the presence of erythrocyte extravasations.

\section{Statistical Analysis}

Results are presented as the mean and standard deviation of the mean (error bars) of at least three independent samples. All statistical evaluations were carried out with unpaired two-tailed Student's $t$-tests. A $p$-value of less than 0.05 was considered to be statistically significant for data-pair comparisons.

\section{RESULTS}

\section{In Vitro Ultrasound Imaging Optimization for MB D/R Modeling}

We first tested the reliability of FUS-induced MB $\mathrm{D} / \mathrm{R}$ in vitro. Typical $\mathrm{B}$-mode imaging contrast changes during the $\mathrm{MB} D / \mathrm{R}$ period, and the corre- sponding mean TIC responses, are shown in Figures 2(a) and (b), respectively. After estimating the perfusion kinetics, the MB D/R kinetics can be represented by mapping the rate constant $\alpha$ (Figure 2(c)). In this case, the sliding window was fixed to be $115 \times 115 \mu \mathrm{m}$ to estimate $\alpha$ from the curve-fitted integrated TIC of the sliding window. This allowed estimation of the 2-D $\alpha$ map. The measured mean rate constant $\alpha$ in this example was $(1.0 \pm 0.2) \mathrm{s}^{-1}$. In addition, the laminar flow effect of MBs could be observed: the $\alpha$ value was larger in the inner than the outer region of the tube, implying a higher MB perfusion rate in the tube center, and decreased perfusion caused by the viscosity of the tube shell. This correlated with traditional physical flow phenomena [28], and confirmed the qualitative correctness of our perfusion kinetics estimation. Thus, the rate constant $\alpha$ map could potentially be used to observe flow and perfusion changes in a small-animal brain. We also confirmed that employed MBs during FUS-induced $D / R$ phase can provide stable and satisfactory TIC estimated quality for the following analyses (see Additional file 1: Supplemental Figure S5).
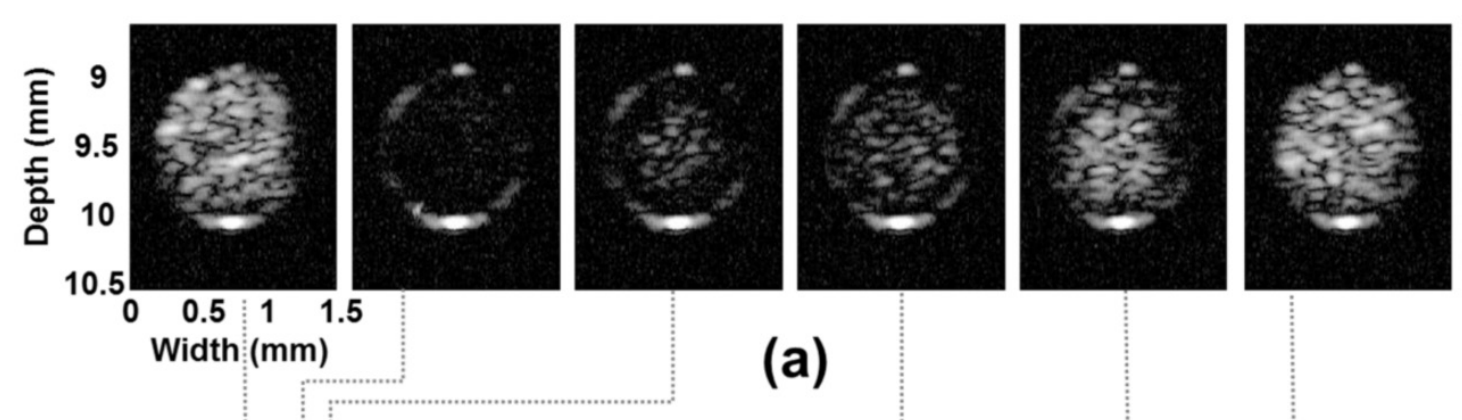
55 (dB) 45

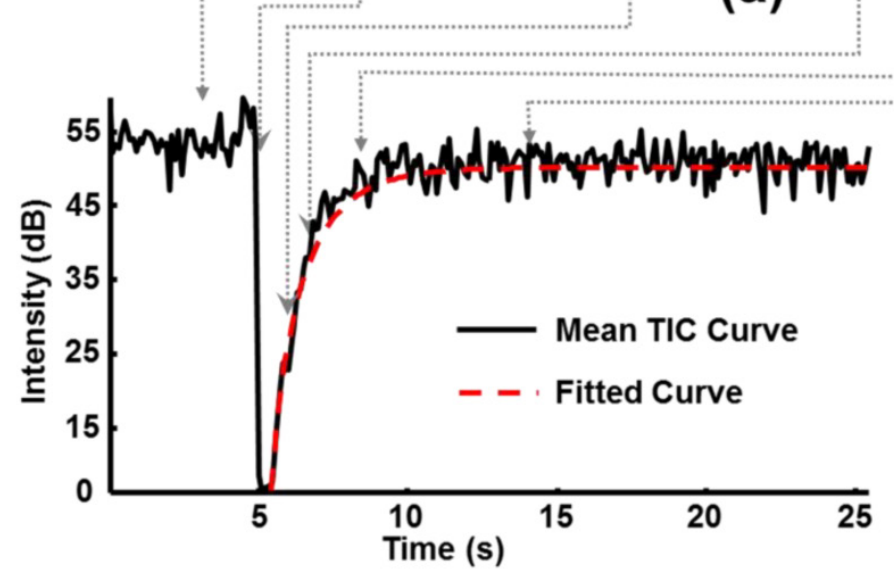

(b)

(c)

Figure 2. Typical in vitro phantom experiment to demonstrate ultrasound perfusion kinetic modeling using the MB D/R approach. (a) Consecutive ultrasound B-mode images of a tube phantom (inner diameter of tube $=1 \mathrm{~mm}$; imaging frequency $=40 \mathrm{MHz}$; $\mathrm{MB}$ destruction frequency $=2 \mathrm{MHz}$ with pressure of $0.6 \mathrm{MPa}$ ). (b) Corresponding mean $\mathrm{TIC}$ obtained from the inner tube region as a ROI. (c) Established rate constant $\alpha$-mapping distribution. 


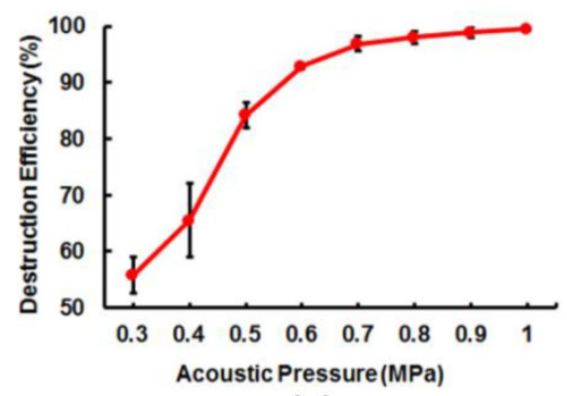

(a)

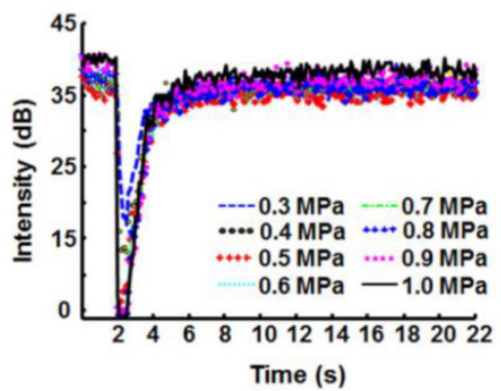

(b)

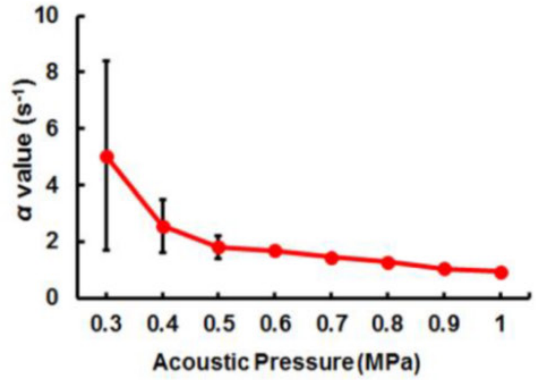

(c)

Figure 3. In vitro phantom experiment to evaluate the influence of pressure of MB destruction on the estimation of kinetic parameters. (a) Efficiency of MB destruction at different acoustic pressures. (b) TIC response at different acoustic pressures. (c) Variation in rate constant $\alpha$-value estimation at different acoustic pressures.

To gain information for the design of further experiments, we measured the $\mathrm{MB}$ activity in response to MB-destroying FUS excitation. MBs started to present apparent broadband emissions at excitation pressures higher than $0.5 \mathrm{MPa}$, and reached their resonant peak when the pressure was increased to 1.0 MPa (Additional file 1: Supplemental Figure S6; for comparison, the tube phantom containing only perfused water did not yield acoustic harmonic emissions). A further increase in ultrasound pressure ( $>1.2$ $\mathrm{MPa}$ ) led to an inverse reduction in MB resonant activity, implying that the majority of the MBs were directly disrupted by the initial ultrasound excitation cycle. This in vitro experiment confirmed that MBs were destroyed at 0.5-1.6 MPa. Within this range of pressure levels, acoustic cavitation (including stable and inertial cavitation) and immediate MB disruption are expected to occur in different proportions, depending on the exact excitation pressure level.

Next we evaluated the acoustic pressure which was required to sufficiently characterize perfusion kinetics. The efficiency of MB destruction reached $(55.8 \pm 3.2) \%,(65.5 \pm 6.6) \%$, and $(84.2 \pm 2.3) \%$ at excitation pressures of $0.3,0.4$, and $0.5 \mathrm{MPa}$, respectively, and $90 \%$ of MBs were destroyed at $0.6 \mathrm{MPa}$ (Figure $3(\mathrm{a})$; burst length $=10$ cycles, $\mathrm{PRF}=4000 \mathrm{~Hz}$, exposure time $=0.5 \mathrm{~s}$, mechanical index $=0.42$ ). In addition, a stable drop in TIC (Figure 3(b)) and a consistent and stable value for the estimated rate constant $\alpha$ (Figure $3(\mathrm{c})$ ) could be acquired at an ultrasound excitation pressure of $0.5 \mathrm{MPa}$.

We also investigated whether TIC dynamics were affected by flow perfusions or MB concentration (Additional file 1: Supplemental Figures S7 (a) and (b)). The estimated rate constant $\alpha$ was found to be sensitive to flow perfusion rate variations from 0.53 to $2.63 \mathrm{~mm} / \mathrm{s}$, which mimicked microvascular flow in vivo. This effect suggested that rate constant $\alpha$ could be used for precise prediction of the perfusion fluid velocity. Estimation of rate constant $\alpha$ was found to be sufficiently robust and independent of MB concentra- tion at the range of $10^{5}$ to $10^{6}$ bubbles $/ \mathrm{mL}$ in circulation (Supplemental Figure S7(c)).

\section{In Vivo Ultrasound Imaging Mapping of MB-FUS-Induced BBB Opening}

Our characterization of in vitro perfusion kinetics provided important information for optimizing the in vivo experimental protocol. An ultrasound pressure of $0.6 \mathrm{MPa}$ was expected to induce a sufficiently high rate of MB destruction to produce stable TIC dynamics and allow estimation of the rate constant $\alpha$. We further verified that FUS exposure reaching $0.5 \mathrm{MPa}$ (burst length $=2000$ cycles; PRF $=1 \mathrm{~Hz}$; exposure time $=60 \mathrm{~s}$; mechanical index $=0.35$ ) induced a BBB opening effect in a small animal (Additional file 1: Supplemental Figure S8), and we demonstrated that MB-destroying emission $(0.6 \mathrm{MPa}$, burst length $=10$ cycles; PRF $=4000 \mathrm{~Hz}$; exposure time $=0.5 \mathrm{~s}$; mechanical index $=0.42$ ) did not induce any changes in BBB integrity (Additional file 1: Supplemental Figure S9). Therefore, the MB destruction pressure was chosen as $0.6 \mathrm{MPa}$, whereas the $\mathrm{BBB}$ opening pressure of the FUS inducer was selected to be $0.5 / 0.7 \mathrm{MPa}$.

A typical in vivo animal experiment for comparison of TICs between FUS-exposed and control normal brain tissues is shown in Figure 4. An in-house built ultrasound imaging and FUS sonication platform was used, with the MB destruction area covering the whole observed brain area (detailed ultrasound specifications provided in Additional file 1: Supplemental Table S1). Regions subjected to BBB opening revealed distinct TIC dynamics compared to the unexposed region. The moderately BBB opening region (region 2) had delayed MB re-perfusion dynamics, whereas the heavy BBB opening region (region 1) presented a further retardation in re-perfusion dynamics compared to control (region 3 ). The rate constant $\alpha$ dropped significantly from its original value of $3.35 \mathrm{~s}^{-1}$ for the control to $1.1 \mathrm{~s}^{-1}$ and $0.19 \mathrm{~s}^{-1}$ in the moderately and heavily BBB opening regions, respectively. We concluded that ultrasound-observed $\mathrm{MB}$ 
reperfusion dynamics could be used to successfully distinguish BBB opening status from TIC response.

Next we attempted high resolution, window-by-window (window size $=825 \times 825 \mu \mathrm{m}$ ) analysis of the rate constant $\alpha$-change map (defined as the difference in the rate constant $\alpha$ before and after FUS-induced BBB opening), using a commercial ultrasound imaging system and FUS sonication setup. Typical results from animal experiments including the EB stained regions and the rate constant $\alpha$-change map are shown in Figure 5. We did not detect any remarkable changes in rate constant $\alpha$-change map in the control animal group (Figure 5(a)). In contrast, a $0.5 \mathrm{MPa}$ exposure successfully created localized BBB opening (Figures 5(b) and (c); ROI enlarged $2 \times$ or $4 \times$, respectively), and the estimated rate constant $\alpha$-change maps correlated well with the BBB opening regions from stained brain sections. Histological examination indicated that a $0.5 \mathrm{MPa}$ exposure did not induce gross pathological changes after inducing BBB opening (Additional file 1: Supplemental Figure S10), whereas a $0.7 \mathrm{MPa}$ exposure created a more profound BBB opening effect and EB penetration (Figures 5(d) and (e)), but also occasionally induced scattered RBC extravasations at the FUS exposure site (detailed occurrence of RBC extravasation induced by 0.5 and 0.7 MPa FUS exposures was shown in Additional file 1: Supplemental Figure S11).

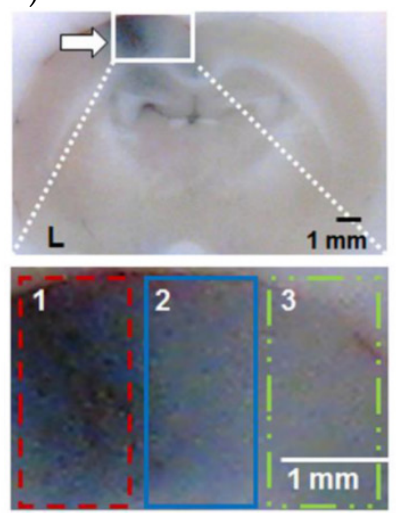

(a)

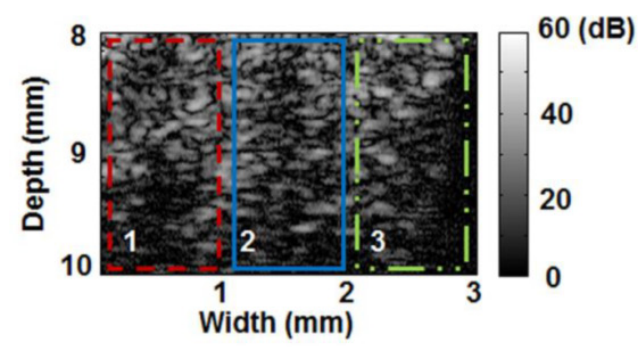

(b)

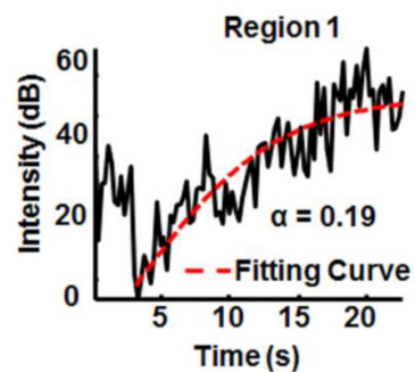

(c)

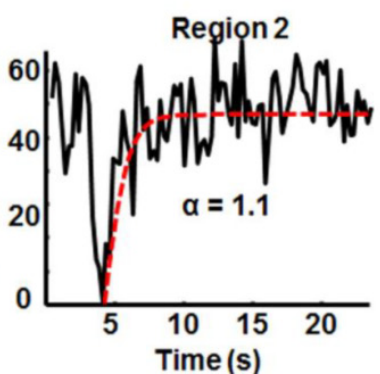

(d)

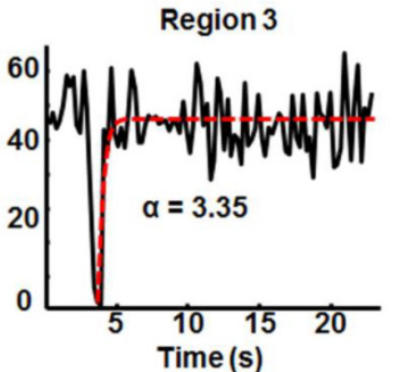

(e)

Figure 4. Typical in vivo animal experiment to estimate the TIC response and corresponding kinetic parameter rate constant $\alpha$ distribution within the selected regions (I: Heavy BBB opening region at the FUS exposure center; 2: mild BBB opening region at the FUS exposure peripheral; 3: normal tissue without FUS exposure). (a) Upper: whole EB dye brain section; lower: $4 \times$ enlarged. (b) Corresponding B-mode images after MBs destruction (corresponding to the lower panel of (a)). (c) Corresponding TIC responses at the three selected regions and the estimated $\alpha$ values. Arrow: BBB-opening region. 


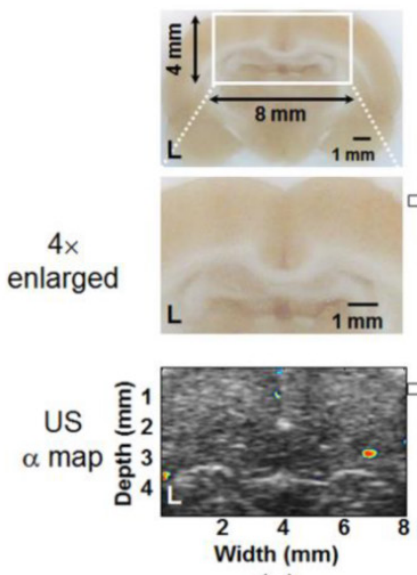

(a)

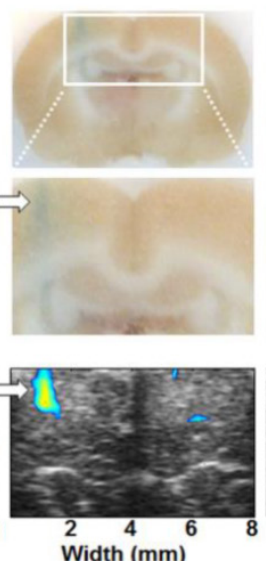

(b)

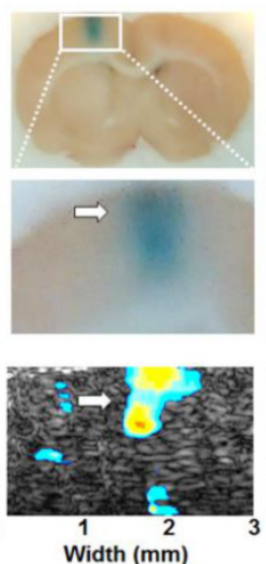

(c)

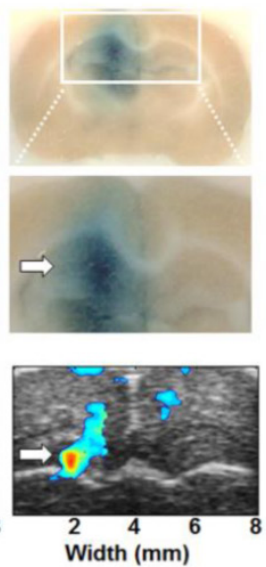

(d)

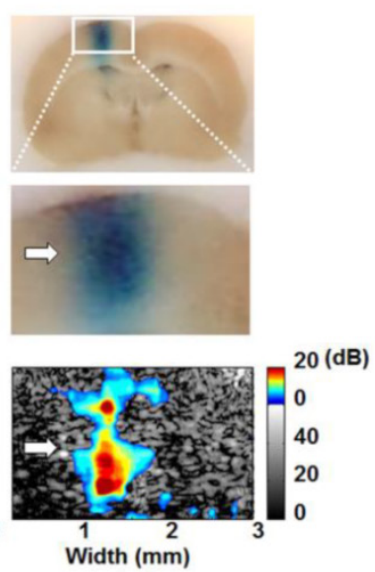

(e)

Figure 5. EB-stained brain sections (upper: original; middle: $2 \times / 4 \times$ enlarged) and corresponding rate constant $\alpha$-change map distributions (bottom panels; $2 \times / 4 \times$ enlarged) to evaluate FUS-induced BBB opening in left hemisphere. (a) Control; (b) and (c) FUS exposure pressure of 0.5 MPa; (d) and (e) FUS exposure pressure of 0.7 MPa. Arrow: BBB-opening region.

\section{Correlation of In Vivo Ultrasound-Monitoring and MRI Detection of BBB Opening}

To verify the reliability and accuracy of $M B D / R$ mapping from ultrasound imaging to monitor $\mathrm{BBB}$ opening, we carried out DCE-MRI on the same experimental animals. Typical results from one control, two $0.5 \mathrm{MPa}$ exposures, and two $0.7 \mathrm{MPa}$ exposures are shown as CE-T1 MR images (to illustrate the signal intensity change due to Gd-DTPA leakage), MR Ktrans mapping (to show kinetic change of blood-to-brain permeability), and estimated ultrasound rate constant $\alpha$-change maps in Figure 6. Control animals showed no contrast changes in CE-T1 MR images, MR Ktrans mapping, or ultrasound rate constant $\alpha$-change maps (Figure 6(a)). BBB opening was induced near the cortical surface of the left hemisphere by $0.5 \mathrm{MPa}$ exposure, and could be clearly detected in both CE-T1 MR images and MR Ktrans distributions (Figures 6(b) and (c)). The corresponding ultrasound rate constant $\alpha$-change map showed a change in contrast at the exposure site, which was co-localized with the MRI signal. A relatively higher contrast change at the left cortical/sub-cortical region was observed for the $0.7 \mathrm{MPa}$ exposure compared to the $0.5 \mathrm{MPa}$ exposure based on CE-T1 MR images and MR Ktrans mapping. The ultrasound rate constant $\alpha$-change map also revealed a change in contrast that was highly correlated with the MR signal (Figures $6(d)$ and (e)). Notably, the ultrasound rate constant $\alpha$-change maps presented a distinct increase in signal from $(8.2 \pm 0.3) \mathrm{dB}$ to $(15.6 \pm 0.5) \mathrm{dB}$ for the 0.5 and 0.7 $\mathrm{MPa}$ exposures, respectively.

The correlation of the ultrasound rate constant $\alpha$-change map with either MR Ktrans mapping or the CE-T1 MR signal change was analyzed (Figure 7). There was a better correlation between $\alpha$-change mapping and MR Ktrans mapping than between the rate constant $\alpha$-change map and the CE-T1 MR signal intensity change $\left(R^{2}=0.84\right.$ and 0.76 , respectively; Figures $7(\mathrm{a})$ and $7(\mathrm{c})$ ). The true-positive $(T P)$, false negative $(F N)$, and false positive $(F P)$ rates were $(89.5$ $\pm 4.8) \%$, $(12.6 \pm 5.4) \%$, and $(40.1 \pm 12.9) \%$, respectively for Ktrans mapping (Figure 7(b)). In other words, when using MR Ktrans mapping as the benchmark, the precision of ultrasound rate constant $\alpha$-change mapping reached $87.6 \%$ (defined as $T P /(T P+F N) \times 100 \%$ ) with sensitivity up to $69.1 \%$ (defined as $T P /(T P+F P) \times 100 \%)$. On the other hand, if using CE-T1 MR signal intensity change as the benchmark, the $T P, F N$, and $F P$ rates were $(87.6 \pm$ $5.3) \%$, $(15.5 \pm 4.8) \%$, and $(68.8 \pm 12.5) \%$, respectively, so that the precision and detection sensitivity were $84.9 \%$ and $56 \%$, respectively (Figure $7(\mathrm{~d})$ ).

\section{Discussion}

In this study, we presented the use of $M B D / R$ modeling to monitor changes in BBB integrity. Specifically, we evaluated the use of rate constant $\alpha$-change mapping to monitor FUS-induced BBB opening. Our data indicated that rate constant $\alpha$-change mapping could indeed provide high detection sensitivity and precision, and was highly correlated with monitoring via MRI. To our knowledge, this is the first demonstration of the successful application of ultrasound imaging to detect FUS-induced BBB opening. Since diagnostic ultrasound has intrinsic benefits including its high temporal resolution and convenience by virtue of being integrated with therapeutic ultrasound, we believe that ultrasound-based detection of FUS-induced BBB opening can provide a useful and valuable alternative to other detection methods in the clinical application of FUS brain drug delivery. 


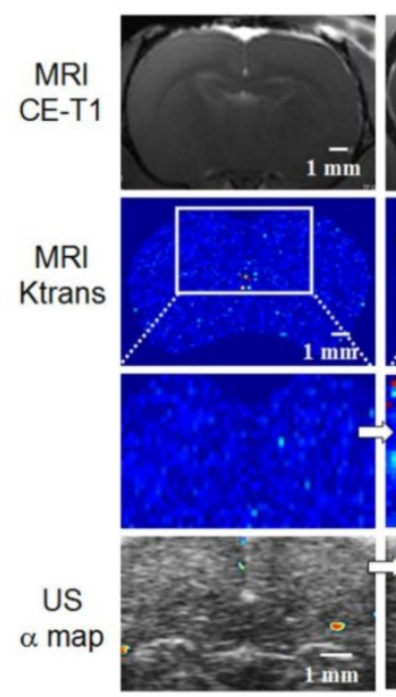

(a)

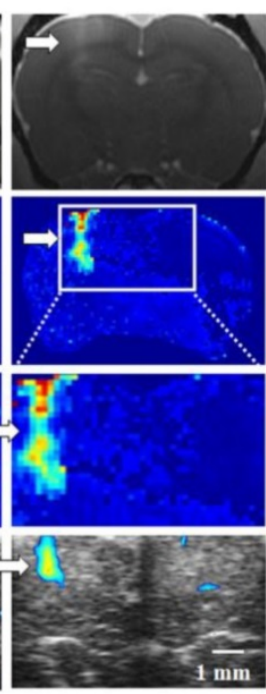

(b)

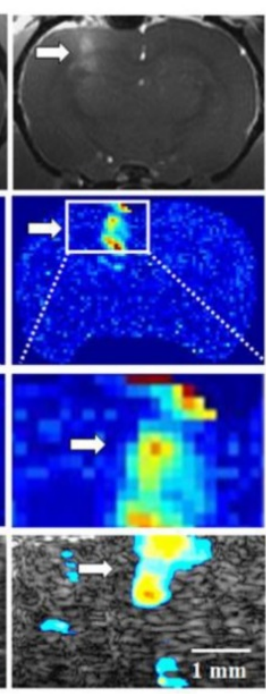

(c)

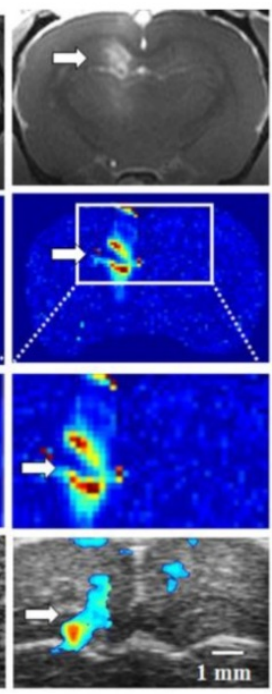

(d)

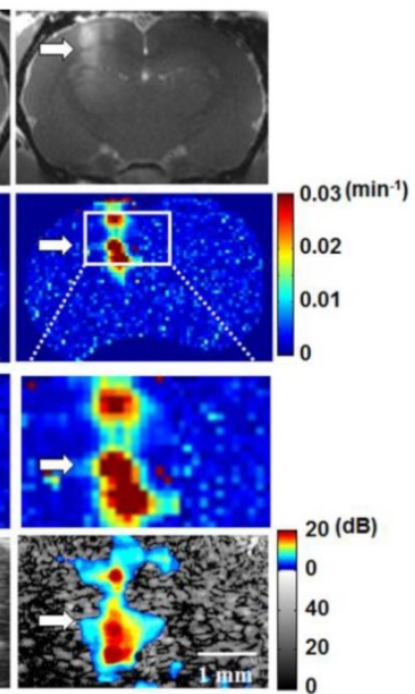

(e)

Figure 6. Comparison of MRI-based detection (upper panels: CE-TI MR images; $2^{\text {nd }}$ and $3^{\text {rd }}$ panels: MR Ktrans map, original and $2 \times / 4 \times$ enlarged) and rate constant $\alpha$-change map (bottom panels; $2 \times / 4 \times$ enlarged) of FUS-induced BBB opening. (a) Control. (b) and (c) FUS exposure pressure of $0.5 \mathrm{MPa}$. (d) and (e) FUS exposure pressure of $0.7 \mathrm{MPa}$. Arrow: BBB-opening region.

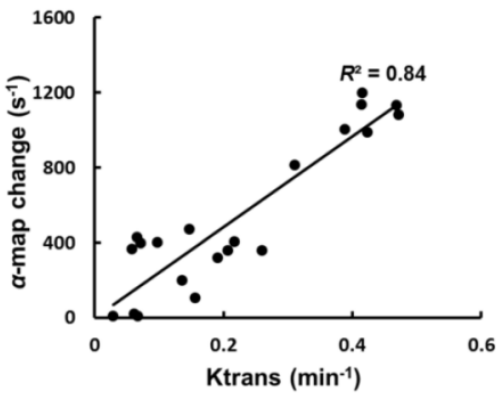

(a)

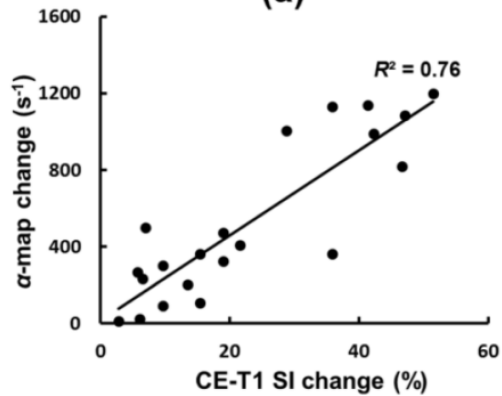

(c)

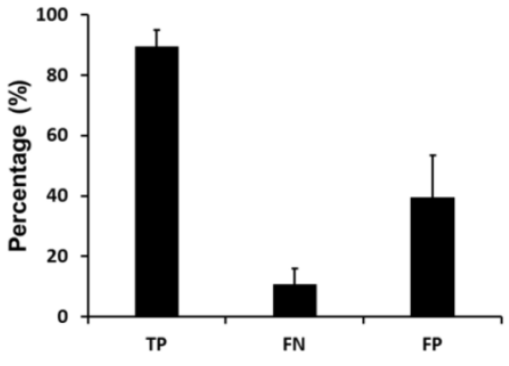

(b)

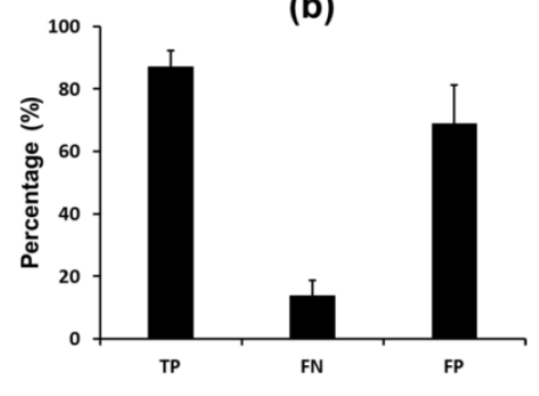

(d)

Figure 7. (a) and (b) Correlation and the corresponding occurrence of true-positive (TP), false negative (FN), and false positive (FP) when comparing rate constant $\alpha$-change map distribution to MR Ktrans map. (c) and (d) Correlation and the corresponding occurrence of TP, FN, and FP when comparing rate-constant $\alpha$-change map distribution to CE-TI MR images.

The MB D/R technique was originally developed to estimate the perfusion of various organs including heart, kidney, liver, and also CNS [21, 28-30]. In this study, we confirmed that the technique can also be applied to monitor FUS-induced BBB opening. This implies that the process of FUS-induced BBB opening correlates with changes in blood flow/ perfusion at the FUS-exposed brain tissues and that this effect persist even 120s after FUS sonication. We previously demonstrated induction of a shortage in blood supply after FUS-induced BBB opening [20]. In addition, fluorescence multi-photon microscopy was recently applied for real-time monitoring of the dynamics of fluorescent dye leakage caused by FUS-induced BBB opening [18, 32]. FUS exposure appears to cause capillaries to experience mechanical stress, thus inducing transient vasoconstrictions and changes in local perfusion. Our real-time fluorescent microscopy observations confirmed that FUS exposure at either 0.5 or $0.7 \mathrm{MPa}$ pressure resulted in vas- 
oconstriction of MB-containing capillaries (Additional file 1: Supplemental Figure S13), similar to previous reports based on multi-photon microscopy [18, 32]. We therefore hypothesized that vasoconstrictions triggered by FUS exposure in the presence of MBs induce transient changes in blood flow, so that ultrasound perfusion kinetics can be used as an indirect and possibly quantitative index to reflect FUS-induced BBB opening.

The mechanism whereby MB-associated FUS excitation induces transient BBB opening is thought to primarily involve widening of tight junctions due to endothelial cell deformation [32,33]. Ultrasound excitation induces repetitive $\mathrm{MB}$ contraction/ expansion within the lumen of the vessel, causing radial propagation of a mechanical wave from the oscillating bubble cloud. The resulting local changes in intra-lumen pressure then induce shear stress on the adjacent capillary lumen wall, finally inducing endothelial cell deformation and loosening of tight junctions. A number of studies have observed that shear stress on the capillary wall can trigger potassium ions $\left(\mathrm{K}^{+}\right)$release from calcium-activated potassium channels on the endothelial cell membrane, leading to cortical spreading depression (CSD) [18, 34]. Gursoy-Ozdemir et al. confirmed that CSD can alter the integrity of the BBB by inducing overexpression of brain matrix metalloproteinases (MMPs) [35]. Our findings appear to support previous reports of a major role of vasoconstriction of capillaries on the observed endothelial cell deformation.

We found that ultrasound perfusion kinetic change mapping technology could be successfully used to monitor the FUS-induced BBB opening process. However, we note that our study had a number of limitations. First, all animals in this study were subjected to a craniotomy (with skull bone thickness of $\sim 1 \mathrm{~mm}$ ) prior to FUS exposure $(2 \mathrm{MHz}$ in this study) and ultrasound imaging $(40 / 21 \mathrm{MHz}$ in this study). This modification posts a challenge when intending to translate the imaging approach to a clinical setting. Human cranial bone thickness is typically 10 $\mathrm{mm}$ and clinical ultrasound imaging frequency ranges from 5-10 MHz. One possibility to overcome this limitation is to further decrease the operating frequency to similar or even lower than the frequency range of a transcranial Doppler device (2 MHz). In addition, Oreilly et al. recently reported installation of a number of wide-band PVDF hydrophones which co-localized with the hemispherical ultrasound phased array element to allow reception of passive acoustic emission during FUS-induced BBB opening process [36]. Notwithstanding their work only allowed one measurement at the focus point of FUS and not to image, their system could potentially also be applied with our proposed scheme for BBB opening. Second, the rate constant $\alpha$-change map was obtained from perfusion kinetics measured prior to and after FUS exposure. Although the change observation from rate constant $\alpha$-change mapping was identical to current contrast-enhanced imaging approaches, neither method can currently provide monitoring of FUS-induced BBB opening with a referenceless manner. Third, MBs had to be administered multiple times, which is inconvenient. Thus a future improvement of our approach would be to perform the MB D/R process together with $\mathrm{MB}$ administration for FUS exposure, as previously suggested [19]. Finally, only very acute BBB permeability change ( $2 \mathrm{~min}$ after FUS sonication) can be detected by our proposed method due to the effect of vasoconstriction would recover with time. Raymond et al. showed that FUS-induced brain vessel vasoconstriction persisted as long as $5 \mathrm{~min}$ (frequency $=1.029 \mathrm{MHz}$; burst length $=10000$ cycles; $\mathrm{PRF}=1 \mathrm{~Hz}$; acoustic pressure $=0.2 \mathrm{MPa}$; exposure time $=45 \mathrm{~s}$; mechanical index $=0.2$ ) [18]. Fan et al. demonstrated that FUS-induced cerebral blood-supply shortage would recover within $2 \mathrm{~h}$ (frequency = $1 \mathrm{MHz}$; burst length: 10000 cycles; PRF = $1 \mathrm{~Hz}$; exposure time $=60 \mathrm{~s}$; acoustic power $=1.1 \mathrm{MPa}$; mechanical index $=1.1$ ) [20]. In conclusion, we proposed the use of ultrasound perfusion kinetic mapping to monitor FUS-induced BBB opening. We demonstrated that the results from this approach correlated highly with the predicted outcome obtained from MRI, with sufficiently high detection sensitivity and precision. Ultrasound imaging has intrinsic benefits including high temporal resolution, accessibility, and compatibility for integration with therapeutic ultrasound. Our approach provides a new opportunity to pursue ultrasound-monitored FUS-induced BBB opening, and could be a potentially valuable alternative for estimating the distribution of drug delivery.

\section{Supplementary Material}

Additional File 1:

Supplemental Tables S1-S2, Supplemental Figures

S1-S13. http:// www.thno.org/v04p1014s1.pdf

\section{Acknowledgements}

We thank the National Science Council of ROC for grants 100-2218-E-182-001, 101-2627-M-007-001, 101-2221-E-182-002-MY, and 3102-2221-E-182-020MY3, as well as National Tsing Hua University for grant 102N2046E1 for funding support. The authors are grateful to the Molecular Imaging Center of Chang Gung Medical Foundation for instrumentation support. 


\section{Conflict of Interest}

The authors declare no conflict of interest.

\section{References}

1. Abbott NJ, Ronnback L, Hansson E. Astrocyte-endothelial interactions at the blood-brain barrier. Nat Rev Neurosci. 2006; 7: 41-53.

2. Ballabh P, Braun A, Nedergaard M. The blood-brain barrier: an overview: structure, regulation, and clinical implications. Neurobiol Dis. 2004; 16: 1-13.

3. Hawkins BT, Davis TP. The blood-brain barrier/neurovascular unit in health and disease. Pharmacol Rev. 2005; 57: 173-85.

4. Rubin LL, Staddon JM. The cell biology of the blood-brain barrier. Annu Rev Neurosci. 1999; 22: 11-28.

5. Chen Y, Liu L. Modern methods for delivery of drugs across the blood-brain barrier. Adv Drug Deliv Rev. 2012; 64: 640-65.

6. Wong HL, Wu XY, Bendayan R. Nanotechnological advances for the delivery of CNS therapeutics. Adv Drug Deliv Rev. 2012; 64: 686-700.

7. Hynynen K, McDannold N, Sheikov NA, Jolesz FA, Vykhodtseva N. Local and reversible blood-brain barrier disruption by noninvasive focused ultrasound at frequencies suitable for trans-skull sonications. Neuroimage. 2005; 24: 12-20.

8. Hynynen $\mathrm{K}$, McDannold N, Vykhodtseva N, Jolesz FA. Noninvasive MR imaging-guided focal opening of the blood-brain barrier in rabbits. Radiology. 2001; 220: 640-46.

9. Liu HL, Hsu PH, Chu PC, Wai YY, Chen JC, Shen CR et al. Magnetic resonance imaging enhanced by superparamagnetic iron oxide particles: usefulness for distinguishing between focused ultrasound-induced blood-brain barrier disruption and brain hemorrhage. J Magn Reson Imaging. 2009; 29: 31-8.

10. Liu HL, Hua MY, Chen PY, Chu PC, Pan CH, Yang HW et al. Blood-brain barrier disruption with focused ultrasound enhances delivery of chemotherapeutic drugs for glioblastoma treatment. Radiology. 2010; 255: 415-25.

11. Liu HL, Hua MY, Yang HW, Huang CY, Chu PC, Wu JS et al. Magnetic resonance monitoring of focused ultrasound/magnetic nanoparticle targeting delivery of therapeutic agents to the brain. Proc Natl Acad Sci U S A. 2010; 107: 15205-10.

12. Wei KC, Tsai HC, Lu YJ, Yang HW, Hua MY, Wu MF et al. Neuronavigation-guided focused ultrasound-induced blood-brain barrier opening: a preliminary study in swine. AJNR Am J Neuroradiol. 2013;34: 115-20.

13. Vlachos F, Tung YS, Konofagou E. Permeability dependence study of the focused ultrasound-induced blood-brain barrier opening at distinct pressures and microbubble diameters using DCE-MRI. Magn Reson Med. 2011;66: 821-30.

14. Vlachos F, Tung YS, Konofagou EE. Permeability assessment of the focused ultrasound-induced blood-brain barrier opening using dynamic contrast-enhanced MRI. Phys Med Biol. 2010;55: 5451-66.

15. Wang PH, Liu HL, Hsu PH, Lin CY, Wang CR, Chen PY et al. Gold-nanorod contrast-enhanced photoacoustic micro-imaging of focused-ultrasound induced blood-brain-barrier opening in a rat model. J Biomed Opt. 2012;17: 061222.

16. Lin KJ, Liu HL, Hsu PH, Chung YH, Huang WC, Chen JC et al. Quantitative micro-SPECT/CT for detecting focused ultrasound-induced blood-brain barrier opening in the rat. Nucl Med Biol. 2009; 36: 853-61.

17. Chen H, Kreider W, Brayman AA, Bailey MR, Matula TJ. Blood vessel deformations on microsecond time scales by ultrasonic cavitation. Phys Rev Lett. 2011; 106: 034301.

18. Raymond SB, Skoch J, Hynynen K, Bacskai BJ. Multiphoton imaging of ultrasound/Optison mediated cerebrovascular effects in vivo. J Cereb Blood Flow Metab. 2007; 27: 393-403.

19. Goertz DE, Wright C, Hynynen K. Contrast agent kinetics in the rabbit brain during exposure to therapeutic ultrasound. Ultrasound Med Biol. 2010;36: 916-24.

20. Fan $\mathrm{CH}$, Liu HL, Huang CY, Ma YJ, Yen TC, Yeh CK. Detection of intracerebral hemorrhage and transient blood-supply shortage in focused-ultrasound-induced blood-brain barrier disruption by ultrasound imaging. Ultrasound Med Biol. 2012; 38: 1372-82.

21. Wei K, Jayaweera AR, Firoozan S, Linka A, Skyba DM, Kaul S. Quantification of myocardial blood flow with ultrasound-induced destruction of microbubbles administered as a constant venous infusion. Circulation. 1998; 97: 473-83.

22. Rim SJ, Leong-Poi H, Lindner JR, Couture D, Ellegala D, Mason $\mathrm{H}$ et al. Quantification of cerebral perfusion with "Real-Time" contrast-enhanced ultrasound. Circulation. 2001; 104: 2582-87.

23. Arditi M, Frinking PJ, Zhou X, Rognin NG. A new formalism for the quantification of tissue perfusion by the destruction-replenishment method in contrast ultrasound imaging. IEEE Trans Ultrason Ferroelectr Freq Control. 2006; 53: 1118-29.

24. Krix M, Kiessling F, Farhan N, Schmidt K, Hoffend J, Delorme S. A multivessel model describing replenishment kinetics of ultrasound contrast agent for quantification of tissue perfusion. Ultrasound Med Biol. 2003; 29: 1421-30.
25. Lucidarme O, Franchi-Abella S, Correas JM, Bridal SL, Kurtisovski E, Berger G. Blood flow quantification with contrast-enhanced US: "entrance in the section" phenomenon--phantom and rabbit study. Radiology. 2003;228: 473-9.

26. Yeh CK, Lu SY, Chen YS. Microcirculation volumetric flow assessment using high-resolution, contrast-assisted images. IEEE Trans Ultrason Ferroelectr Freq Control. 2008; 55: 74-83.

27. Tofts PS, Brix G, Buckley DL, Evelhoch JL, Henderson E, Knopp MV et al. Estimating kinetic parameters from dynamic contrast-enhanced T(1)-weighted MRI of a diffusable tracer: standardized quantities and symbols. J Magn Reson Imaging. 1999; 10: 223-32.

28. Albrecht T, Hoffmann CW, Schettler S, Overberg A, Ilg M, von Behren PL et al. B-mode enhancement at phase-inversion US with air-based microbubble contrast agent: initial experience in humans. Radiology. 2000; 216: 273-8.

29. Guibal A, Lefort T, Chardon L, Benslama N, Mule S, Pilleul F et al. Contrast-enhanced ultrasound after devascularisation of neuroendocrine liver metastases: functional and morphological evaluation. Eur Radiol. 2013;23: $805-15$.

30. Kalantarinia K, Belcik JT, Patrie JT, Wei K. Real-time measurement of renal blood flow in healthy subjects using contrast-enhanced ultrasound. Am J Physiol Renal Physiol. 2009; 297: 1129-34.

31. Kern R, Diels A, Pettenpohl J, Kablau M, Brade J, Hennerici MG et al. Real-time ultrasound brain perfusion imaging with analysis of microbubble replenishment in acute MCA stroke. J Cereb Blood Flow Metab. 2011;31: 1716-24.

32. Sheikov N, McDannold N, Sharma S, Hynynen K. Effect of focused ultrasound applied with an ultrasound contrast agent on the tight junctional integrity of the brain microvascular endothelium. Ultrasound Med Biol. 2008; 34: 1093-104.

33. Sheikov N, McDannold N, Vykhodtseva N, Jolesz F, Hynynen K. Cellular mechanisms of the blood-brain barrier opening induced by ultrasound in presence of microbubbles. Ultrasound Med Biol. 2004; 30: 979-89.

34. Koroleva VI, Vykhodtseva NI, Elagin VA. Spreading depression in the cortex and subcortical structures of the brain of the rat induced by exposure to focused ultrasound. Neirofiziologiia. 1986; 18: 55-61.

35. Gursoy-Ozdemir Y, Oiu J, Matsuoka N, Bolay H, Bermpohl D, Jin $\mathrm{H}$ et al. Cortical spreading depression activates and upregulates MMP-9. J Clin Invest. 2004; 113: 1447-55.

36. O'Reilly MA, Hynynen K. A PVDF receiver for ultrasound monitoring of transcranial focused ultrasound therapy. IEEE Trans Biomed Eng. 2010; 57: 2286-94. 